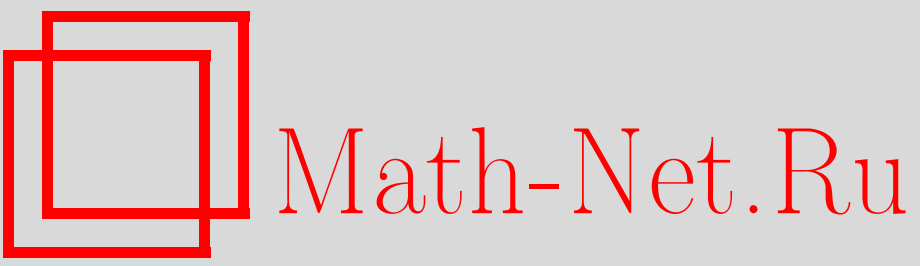

С. П. Суетин, О теореме Дюма в теории непрерывных дробей, УМН, 2002, том 57, выпуск 5, 163-164

DOI: https://doi.org/10.4213/rm566

Использование Общероссийского математического портала Math-Net.Ru подразумевает, что вы прочитали и согласны с пользовательским соглашением

http://www.mathnet.ru/rus/agreement

Параметры загрузки:

IP : 54.237 .206 .68

26 апреля 2023 г., 08:38:42 


\section{О ТЕОРЕМЕ ДЮМА В ТЕОРИИ НЕПРЕРЫВНЫХ ДРОБЕЙ}

\section{С. П. Суетин}

1. В теории непрерывных дробей хорошо известна теорема Дюма [1] (см. также [2]) о поведении чебышевской непрерьвной дроби [3] для квадратного корня из полинома четвертой степени с простыми комплексными корнями. Точнее, пусть функция $f$ имеет вид

$$
f(z)=\sqrt{\left(z-e_{1}\right) \cdots\left(z-e_{4}\right)}-z^{2}+\frac{e_{1}+\cdots+e_{4}}{2} z,
$$

где точки $e_{1}, \ldots, e_{4} \in \mathbb{C}$ попарно различны и выбрана та ветвь корня, главный член которой в окрестности бесконечно удаленной точки $z=\infty$ равен $z^{2}$; тем самым, $f \in \mathscr{H}(\infty)$. Как известно, $n$-я подходящая дробь $C_{n}$ есть в точности $n$-я диагональная аппроксимация Паде $[n / n]_{f}$ функции $f$ в бесконечно удаленной точке. Напомним, что $[n / n]_{f}=P_{n} / Q_{n}$, где $P_{n}, Q_{n} \in \mathbb{C}[z]$, $\operatorname{deg} P_{n}, \operatorname{deg} Q_{n} \leqslant n, Q_{n} \not \equiv 0$ и $\left(Q_{n} f-P_{n}\right)(z)=O\left(z^{-n-1}\right), z \rightarrow \infty$. В соответствии с общей теоремой Шталя [4] о сходимости аппроксимаций Паде для алгебраических функций, для функции вида (1) имеем: последовательность $[n / n]_{f}$ сходится $\kappa f$ по (логарифмической) емкости внутри области $D=\overline{\mathbb{C}} \backslash S$, где $S$ - компакт минималшной емкости [5], [6], соответствующий алгебраической функции $\sqrt{\left(z-e_{1}\right) \cdots\left(z-e_{4}\right)}$ : выбранная вьше ветвь корня (а вместе с ней и функция $f$ ) продолжается из окрестности точки $z=\infty$ в дополнение к $S$ как однозначная аналитическая функция, а из всех компактов, обладающих таким свойством, $S$ имеет наименьшую емкость.

В [1] Дюма исследовал вопрос о равномерной сходимости последовательности подходящих дробей $\left\{C_{n}\right\}$ в предположении, что $S$ состоит в точности из двух (непересекающихся) дуг: $S=$ $S_{1} \cup S_{2}$. В частности, он показал, что если определенные числовые параметры, связанные толшко с расположением точек $e_{1}, \ldots, e_{4}$, рационально независимы, то множество полюсов подходящих дробей $C_{n}$ всюду плотно в $\overline{\mathbb{C}}$, т.е. последовательность $\left\{C_{n}\right\}$ не может равномерно сходиться ни в какой подобласти $D$. Поскольку $C_{n}=[n / n]_{f}$, то резулштат Дюма о поведении полюсов подходящих дробей есть фактически утверждение об асимптотике знаменателей $Q_{n}$. Полиномш $Q_{n}$, как известно, ортогоналшны на $S=S_{1} \cup S_{2}$ с весом $\sqrt{\left(\zeta-e_{1}\right) \cdots\left(\zeta-e_{4}\right)}, \zeta \in S$; если, в частности, все $e_{1}, \ldots, e_{4} \in \mathbb{R}$, то $S$ состоит из двух отрезков, а $Q_{n}$ - полиномы Ахиезера [7].

Для функции $\sqrt{z^{2}-1}-z \in \mathscr{H}(\infty)$ знаменатели подходящих дробей - полиномш Чебьшёва II-го рода, ортогональные на отрезке $[-1,1]$ с весом $\sqrt{1-x^{2}}$. Аналогично, разложение функции $1 / \sqrt{z^{2}-1}$ в непрерывную дробь приводит к полиномам Чебышёва, ортогональным с весом $1 / \sqrt{1-x^{2}}$. Наконец, для функции вида

$$
\frac{1}{\pi} \int_{-1}^{1} \frac{\rho(x)}{\sqrt{1-x^{2}}} \frac{d x}{z-x}
$$

где $\rho \neq 0$, соответствующие полиномы $Q_{n}$ ортогональны на $[-1,1]$ относительно $\rho(x) / \sqrt{1-x^{2}}$ (см. [3]). При условии, что $\rho$ удовлетворяет условию Дини-Липшица, "силњная" асимптотика полиномов $Q_{n}$ была изучена С. Н. Бернштейном (см. [8], а также [9; гл. ХІІ]) с помощью разработанного им параллельно с Г. Сегё аппроксимационного метода.

По аналогии с классической ситуацией мы рассматриваем здесь класс функций вида

$$
\widehat{\rho}(z)=\frac{1}{\pi i} \int_{S} \frac{\rho(\zeta)}{\sqrt{\left(\zeta-e_{1}\right) \cdots\left(\zeta-e_{4}\right)}} \frac{d \zeta}{\zeta-z},
$$

где под $\sqrt{\left(\zeta-e_{1}\right) \cdots\left(\zeta-e_{4}\right)}, \zeta \in S$, понимаются граничные значения на $S$ выбранной в $D$ ветви корня; комплекснозначная функция $\rho(\zeta) \neq 0$ и удовлетворяет условию Дини-Липшица; такую функцию $\rho$ в дальнейшем будем назьвать допустимым весом. Если $\rho \in \mathbb{C}(\zeta)$ и не имеет на $S$ полюсов, то $\widehat{\rho}$ - эллиптическая функция: $\widehat{\rho}(z)=r_{1}(z)+r_{2}(z) / \sqrt{\left(z-e_{1}\right) \cdots\left(z-e_{4}\right)}$, где $r_{1}, r_{2} \in \mathbb{C}(z)$.

Работа выполнена при поддержке Российского фонда фундаментальных исследований (грант № 02-01-00564), Программы поддержки ведущих научных школ России (грант № 00-15-96132) и фонда INTAS (грант № 2000-272). 
Оказьвается, поведение последовательности диагональных аппроксимаций Паде для функций

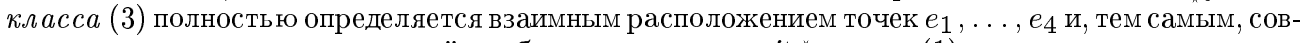
падает с поведением непрерывной дроби для конкретной функции (1).

2. Пусть $g(z, \infty)$ - функция Грина для области $D$ с особенностью в точке $z=\infty, \widetilde{g}(z, \infty)$ гармонически сопряженная к ней функция, $G(z, \infty)=g(z, \infty)+i \widetilde{g}(z, \infty)$ - комплексная функция Грина. Область $D$ - двусвязна, поэтому $G$-многозначная функция. Все периоды функции $G-$ чисто мнимые, причем лишь два из них независимы. В качестве одного периода можно выбрать величину $2 \pi i \omega_{1}(\infty)$, где $\omega_{1}(z)=\omega\left(S_{1}, z ; D\right)$ - гармоническая мера дуги $S_{1}$ в точке $z \in D$ относительно области $D, 0<\omega_{1}(z)<1$. Обозначим другой период через $2 \pi i \Delta_{1}(\infty), \Delta_{1}(\infty) \in \mathbb{R}$. Из $[10$; формула (26)] вытекает, что два вещественных параметра, введенные Дюма для характеризации расположения точек $e_{1}, \ldots, e_{4}$, совпадают с $\Delta_{1}(\infty)$ и $\omega_{1}(\infty)$.

Теорема. Пусть (как и в теореме Дюма) множсество $S$ состоит из двух непересекающих дуг. Тогда справедливы следующие утверждения.

1) Если величины $\Delta_{1}(\infty), \omega_{1}(\infty)$ и 1 рационально независимь, то для любого допустимого веса $\rho$ полюсы аппроксимаций Паде $[n / n]_{\widehat{\rho}}$ всюду плотны в $\overline{\mathbb{C}}$, т.е. последовательность $\left\{[n / n]_{\widehat{\rho}}\right\}$ не может равномерно сходиться ни в какой области $D^{\prime} \Subset D$.

2) Eсли $\Delta_{1}(\infty), \omega_{1}(\infty) \in \mathbb{Q}$, то для любого допустимого веса $\rho$ существует конечное множество точек $Z \subset D$ таких, что множество предельных точек полюсов $[n / n]_{\widehat{\rho}}$ совпадает с $S \cup Z$, а аппроксимации Паде $[n / n]_{\widehat{\rho}}$ сходятся $\kappa \widehat{\rho}$ равномерно внутри области $D \backslash Z$.

3) Если величинь $\Delta_{1}(\infty), \omega_{1}(\infty)$ и 1 рачионально зависимь, но хотя бы одна из величин $\Delta_{1}(\infty), \omega_{1}(\infty)$ - иррациональна, то для любого допустимого веса $\rho$ существует конечное число непересекающихся открытых дуг $\gamma_{1}, \ldots, \gamma_{k} \subset D$, концы которьх принадлежат $S_{1}$ и $S_{2}$, таких, что множество предельных точек полюсов $[n / n]_{\widehat{\rho}}$ совпадает с $S \cup \gamma_{1} \cup \cdots \cup \gamma_{k}$, а аппроксимачии Паде $[n / n]_{\widehat{\rho}}$ сходятся $к \widehat{\rho}$ равномерно внутри области $D \backslash \bigcup_{j=1}^{k} \gamma_{j}$.

Доказательство этого резултата проводится по следующей схеме, впервые реализованной в [10] (см. также [11]) для случая гиперэллиптических функций: 1) существует обладающее специалными свойствами решение проблемы Якоби обращения абелевых интегралов $\Rightarrow 2$ ) существует решение определенной краевой задачи Римана $\Rightarrow 3$ ) выполняется сингулярное интегралное уравнение на ортогональные полиномы $\Rightarrow 4$ ) существует формула для сильной асимптотики ортогональных полиномов $\Rightarrow 5$ ) имеет место равномерная сходимость диагональных аппроксимаций Паде.

ЗАмечАния. І. Для допустимых весов естественньп образом [10] вводятся классы эквивалентности. Множество $Z$ в 2) и набор дуг $\left\{\gamma_{j}\right\}$ в 3) зависят лишь от этих классов.

II. Приведенные резултаты остаются справедливыми: 1) для более общего случая ортогональности с весом $\rho(\zeta) P(\zeta) / \sqrt{\left(\zeta-e_{1}\right) \cdots\left(\zeta-e_{4}\right)}$, где $P \in \mathbb{C}[z]$ и делит полином $\left(z-e_{1}\right) \times$ $\left.\cdots\left(z-e_{4}\right) ; 2\right)$ для функций вида $\widehat{\rho}+r$, где $r \in \mathbb{C}(z) \cap \mathscr{H}(S), r(\infty)=0$; в последнем случае равномерная сходимость аппроксимаций Паде подразумевается в сферической метрике.

\section{СПИСОК ЛИТЕРАТУРЫ}

[1] S. Dumas. Sur le développement des fonctions elliptiques en fractions continues. Thesis. Zürich, 1908. [2] H. Stahl // Ann. Fac. Sci. Toulouse. Math. (6). Spec. Iss. 1996. P. 121-193. [3] П. Л. Чебышёв // Полное собрание сочинений. Т. II. М.-Л.: Изд-во АН СССР, 1948. C. 103-126; 336-341. [4] H. Stahl // Constr. Approx. 1986. V. 2. P. 225-240; 241-251. [5] J. Nuttall // J. Approx. Theory. 1984. V. 42. P. 299-386. [6] H. Stahl // Complex Variables Theory Appl. 1985. V. 4. Р. 311-324; 325-338; 339-354. [7] Н. И. Ахиезер // Докл. АН СССР. 1960. Т. 134. № 1. С. 9-12. [8] С. Н. Бернштейн. О многочленах, ортогональных в конечном интервале. Харьков: ОНТИ, 1937. [9] Г. Сегё. Ортогональные многочлены. М.: Физматгиз, 1962. [10] С. П. Суетин // Матем. сб. 2000. Т. 191. № 9. С. 81-114. [11] С. П. Суетин // УМН. 2002. Т. 57. №1. С. 45-142.

Математический институт

Принято редколлегией

им. В.А. Стеклова РАН

12.09.2002

E-mail: suetin@mi.ras.ru 